\title{
Protective efficacy of a live, attenuated, influenza virus vaccine ('Alice' strain)
}

\author{
A. PRINZIE* \\ M.D. \\ C. HuYgelen $\dagger$ \\ D.V.M. \\ A. Delem $\dagger$ \\ B.Sc. \\ Belgium, and † Recherche et Industrie Thérapeutiques, B-1320 Genval, Belgium
}

\section{Summary}

Animal studies have indicated the high degree of efficacy and broad protection of 'Alice' vaccine against various heterologous $\mathrm{H}_{3} \mathrm{~N}_{2}$ influenza virus strains.

Similarly, challenge studies carried out in volunteers have confirmed the high degree of efficacy of 'Alice' vaccine versus homologous and heterologous influenza A virus strains.

\section{Introduction}

Recent work on influenza immunization has indicated that the most often quoted criterion for successful immunization with a viral vaccine, namely the elevation of circulating serum antibodies, did not correlate completely with protection from overt influenza infection.

In fact, killed influenza vaccines administered parenterally are usually able to induce circulating haemagglutination-inhibition (HI) antibodies, often in close to $100 \%$ of cases, whereas their protective efficacy is accepted in the range of $60-80 \%$. On the contrary, the use of live intranasal vaccines has led to somewhat lower seroconversion rates or geometric mean titres and to higher protective efficacy.

This is exemplified in a comparative trial reported by Beare et al. (1968), in which ninety-eight volunteers were vaccinated with inactivated influenza B virus parenterally or with live influenza $B$ virus intranasally. Seroconversion was observed in $71 \%$ of those given the killed vaccine and in only $51 \%$ of those receiving the live vaccine. These subjects were subsequently challenged with live influenza virus, with the results that influenza infection was reduced by $62 \%$ and $94 \%$ for the killed and live vaccines respectively (Table 1 ).

In another paper, Beare (1969) observed that some subjects vaccinated intranasally with a live influenza vaccine did not develop $\mathrm{HI}$ or neutralizing circulating antibodies and yet could not apparently be reinfected when challenged subsequently. He concluded that the effects of live influenza vaccines were
TABLE 1. Serological response and protection versus challenge

\begin{tabular}{lccc}
\hline & & \multicolumn{2}{c}{ Protection } \\
\cline { 3 - 4 } $\begin{array}{c}\text { Vaccine } \\
\text { type }\end{array}$ & $\begin{array}{c}\text { Serological } \\
\text { HI response }\end{array}$ & $\begin{array}{c}\text { Clinical } \\
\text { symptoms }\end{array}$ & $\begin{array}{c}\text { 'Flu infection } \\
\text { (lab. tests) }\end{array}$ \\
\hline $\begin{array}{l}\text { Killed } \\
\text { vaccine* }\end{array}$ & $71 \%$ & $50 \%$ & $62 \%$ \\
$\begin{array}{l}\text { Live } \\
\text { vaccine* }\end{array}$ & $51 \%$ & $82 \%$ & $94 \%$ \\
\hline
\end{tabular}

* Influenza B/England/13/65.

evidently not to be measured solely by the stimulation of circulating antibodies.

Mann et al. (1968) have also reported that intranasal live influenza virus vaccines seem to be able to afford protection even without stimulation of serum antibody. These results indicate clearly that local infection is superior to parenteral immunization in inducing immunity in respiratory secretions and in providing protection at the site of infection.

It has therefore been postulated that a live attenuated influenza vaccine administered intranasally, by mimicking an asymptomatic natural infection, would stimulate a full immune response and thereby provide a protection similar to that observed after a natural infection.

\section{Material and methods}

The protective efficacy of a live attenuated influenza vaccine ('Alice' strain) has been tested in an animal model and in volunteers, by the use of a challenge technique rather than by the measurement of circulating antibodies. 'Alice' strain has been extensively investigated (Huygelen et al., 1973; Peetermans et al., 1973; Lamy et al., 1973; Peetermans, Lamy and Delem, 1975; Waldman et al., 1975; Rubin et al., 1974; Noble et al., 1974; Prévost et al., 1975 ) and shown to be attenuated, safe and immunogenic. 


\section{Results}

(1) Experiments in ferrets

Groups of ferrets were immunized intranasally with 'Alice' vaccine (one dose containing $10^{6 \cdot 5}-10^{7 \cdot 0}$ $\mathrm{EID}_{50}$ ) and others were kept as controls (Table 2). All animals were challenged 5 weeks later with various influenza virus strains, representative of three recent antigenic drifts.

TABLE 2. Heterologous challenges of ferrets immunized with 'Alice' influenza strain

\begin{tabular}{ll}
\hline \multicolumn{1}{c}{ Vaccine strain } & Challenge strains \\
\hline 'Alice' & A/Dunedin/4/73 \\
serotype A/England/42/72 & (Port Chalmers-like) \\
& A/England/635/74 \\
& A/Scotland/840/74 \\
\hline
\end{tabular}

The parameters used in evaluating protection in these animals are shown in Table 3. Basically there are three criteria: clinical: body temperature and symptoms; viral excretion; nasal protein increase.

TABLE 3. Parameters of protection evaluation

\begin{tabular}{|c|c|c|}
\hline & Body temperature & $\begin{array}{l}\text { One or two readings } \geqslant 40^{\circ} \mathrm{C} \text { at } \\
24-72 \mathrm{hr} \text { post-inoculation } \\
\text { Two readings } \geqslant 1^{\circ} \mathrm{C} \text { above the } \\
\text { mean pre-inoculation tempera- } \\
\text { ture }\end{array}$ \\
\hline (2) & Symptoms & $\begin{array}{l}\text { Depression } \\
\text { Dyspnoea } \\
\text { Nasal discharge } \\
\text { Coughing } \\
\text { Conjunctivitis }\end{array}$ \\
\hline (3) & Viral excretion & Tested $2-4$ days post-inoculation \\
\hline (4) & $\begin{array}{l}\text { Nasal protein } \\
\text { increase on day } 7\end{array}$ & $\begin{array}{l}\text { At least three-fold above the } \\
\text { pre-inoculation level }\end{array}$ \\
\hline
\end{tabular}

As shown in Table 4, there was a complete protection against $\mathrm{A} /$ Dunedin virus (a Port Chalmers-like strain) in all three immunized ferrets, as compared to controls. After challenge with A/England/635 there was an apparent reinfection in two out of three ferrets: one case showing a body temperature increase, another shedding virus on day 2 . However, these signs of infection were much less pronounced than in the controls, for both of these parameters After challenge with A/Scotland virus, one ferret shed virus on day 2 but at a significantly lower titre than the controls.

The response to challenge with England/635 of Scotland/840 was not significantly different from the response observed with Dunedin $/ 4$, indicating that the degree of protection provided by 'Alice' vaccire was about equal against all three $\mathrm{H}_{3} \mathrm{~N}_{2}$ subtypes.

In conclusion, the study in ferrets confirms the broad protection provided by a live influenza virus vaccine (McLarren, Potter and Jennings, 1974).

\section{(2) Experiment in man}

The results of four artificial challenge studies a summarized in Table 5. In total, twenty-two subject. immunized with 'Alice' strain and twenty-eight con' trols were infected with an homologous $\mathrm{H}_{3} \mathrm{~N}_{2}$ virig strain, 3-4 months after vaccination. Several paræ meters were followed:

Illness: on the basis of recorded respiratorn symptoms, protection was observed in $88.5 \%$ of the cases. However, this clinical evaluation did not take into account that some symptoms might not have been associated with influenza.

Influenza infection: when considering influen $\overrightarrow{z a}$ infection as defined either by virus isolation or four-fold rise of antibodies, protection against etpit firmed influenza was observed in $100 \%$ of the cases

Table 6 shows the results of studies still in progress where the challenge virus was either homologous heterologous (Port Chalmers-like). In both case? protection was complete against virus associate? illness.

The evaluation of protective efficacy can also be calculated from natural challenge conditions, i.et evaluating protection after an influenza outbreak a community population containing vaccinees and control subjects. Table 7 illustrates the results of suef a natural challenge. An outbreak of Port Chalmers influenza occurred in a high-risk population, vaces nated 3-4 months previously. There was no difference in the overall rate of illness among 'Alice' vaccinees. intramuscular vaccinees or controls. However, the

TABLE 4. Results of heterologous challenge of ferrets vaccinated intranasally with the 'Alice' strain

\begin{tabular}{|c|c|c|c|c|c|c|c|c|}
\hline \multirow[b]{2}{*}{ Challenge strains } & \multirow[b]{2}{*}{ Group } & \multicolumn{2}{|c|}{ Body temperature } & \multirow[b]{2}{*}{ Symptoms } & \multicolumn{3}{|c|}{ Viral shedding } & \multirow{2}{*}{$\begin{array}{c}\text { Nasal protein } \\
\text { mean increase } \\
\text { on day } 7 \text {. }\end{array}$} \\
\hline & & $\geqslant 40^{\circ} \mathrm{C}$ & $\geqslant 1^{\circ} \mathrm{C}$ & & Day 2 & Day 3 & Day 4 & \\
\hline \multirow{4}{*}{$\begin{array}{l}\text { A/Dunedin } / 4 / 73 \\
\text { (Port Chalmers-like) } \\
\text { A/England } / 635 / 74\end{array}$} & Vaccinees & $0 / 3$ & NT & $0 / 3$ & $0 / 3$ & $0 / 3$ & $0 / 3$ & \multirow[t]{2}{*}{ NS } \\
\hline & Controls & $4 / 4$ & NT & $4 / 4$ & $4 / 4$ & $4 / 4$ & $4 / 4$ & \\
\hline & Vaccinees & $1 / 3$ & $0 / 3$ & None & $1 / 3$ & $0 / 3$ & $0 / 3$ & \multirow{2}{*}{ 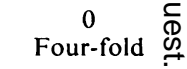 } \\
\hline & Controls & $3 / 3$ & $1 / 3$ & None & $3 / 3$ & $3 / 3$ & $3 / 3$ & \\
\hline \multirow[t]{2}{*}{ A/Scotland $/ 840 / 74$} & Vaccinees & $0 / 2$ & $0 / 2$ & $0 / 2$ & $1 / 2$ & $0 / 2$ & $0 / 2$ & \multirow[t]{2}{*}{ NS } \\
\hline & Controls & $2 / 3$ & $2 / 3$ & $3 / 3$ & $3 / 3$ & $3 / 3$ & $2 / 3$ & \\
\hline
\end{tabular}


TABLE 5. Challenge studies

\begin{tabular}{|c|c|c|c|c|c|c|c|c|}
\hline \multirow[b]{2}{*}{ Study groups } & \multirow[b]{2}{*}{$\begin{array}{l}\text { Challenge virus } \\
\text { dose/person }\end{array}$} & \multirow[b]{2}{*}{ Illness } & \multirow[b]{2}{*}{$\begin{array}{l}\text { Virus } \\
\text { isolation }\end{array}$} & \multirow[b]{2}{*}{$\begin{array}{l}\text { Serum } \\
4 \mathrm{x} \text { rise }\end{array}$} & \multirow[b]{2}{*}{$\begin{array}{l}\text { Nasal } \\
4 \mathrm{x} \text { rise }\end{array}$} & \multirow{2}{*}{$\begin{array}{l}\text { Virus } \\
\text { associated } \\
\text { illness }\end{array}$} & \multicolumn{2}{|c|}{ Protection rate $(\%)$} \\
\hline & & & & & & & Illness & $\begin{array}{c}\text { Virus } \\
\text { associated }\end{array}$ \\
\hline $\begin{array}{l}\text { (1) 'Alice'-2 doses } \\
\text { Control }\end{array}$ & $\begin{array}{l}\text { Udorn BEK } 4 \\
10^{2 \cdot 12} \text { TCID }_{50}\end{array}$ & $\begin{array}{l}1 / 6 \\
6 / 11\end{array}$ & $\begin{array}{l}1 / 6 \\
6 / 11\end{array}$ & $\begin{array}{l}1 / 6 \\
3 / 11\end{array}$ & $\begin{array}{l}1 / 6 \\
0 / 11\end{array}$ & $\begin{array}{l}\text { None } \\
5 / 11\end{array}$ & 69 & 100 \\
\hline $\begin{array}{l}\text { (2) 'Alice'-2 doses } \\
\text { Control }\end{array}$ & $\begin{array}{l}\text { A/Engl/42/72 RIT } \\
10^{6.8} \mathrm{EID}_{50}\end{array}$ & $\begin{array}{l}0 / 4 \\
1 / 5\end{array}$ & $\begin{array}{l}3 / 4 \\
4 / 5\end{array}$ & $\begin{array}{l}1 / 4 \\
1 / 5\end{array}$ & $\begin{array}{l}- \\
-\end{array}$ & $\begin{array}{l}\text { None } \\
1 / 5\end{array}$ & 100 & 100 \\
\hline $\begin{array}{l}\text { (3) 'Alice'-2 doses } \\
\text { Control }\end{array}$ & $\begin{array}{l}\text { A/Engl/42/72 RIT } \\
10^{6.8} \mathrm{EID}_{50}\end{array}$ & $\begin{array}{l}0 / 6 \\
1 / 6\end{array}$ & $\begin{array}{l}0 / 6 \\
1 / 6\end{array}$ & $\begin{array}{l}0 / 6 \\
4 / 6\end{array}$ & $\begin{array}{l}3 / 6 \\
4 / 6\end{array}$ & $\begin{array}{l}\text { None } \\
1 / 6\end{array}$ & 100 & 100 \\
\hline $\begin{array}{l}\text { (4) 'Alice'-2 doses } \\
\text { Control }\end{array}$ & $\begin{array}{l}\text { Udorn BEK4E1 RIT } \\
10^{6 \cdot 12} \mathrm{EID}_{50}\end{array}$ & $\begin{array}{l}0 / 6 \\
3 / 6\end{array}$ & $\begin{array}{l}0 / 6 \\
4 / 6\end{array}$ & $\begin{array}{l}0 / 6 \\
4 / 6\end{array}$ & $\begin{array}{l}0 / 6 \\
4 / 6\end{array}$ & $\begin{array}{l}\text { None } \\
3 / 6\end{array}$ & 100 & 100 \\
\hline $\begin{array}{l}\text { Total 'Alice' } \\
\text { Control }\end{array}$ & & $\begin{array}{r}1 / 22 \\
11 / 28\end{array}$ & $\begin{array}{r}4 / 22 \\
15 / 28\end{array}$ & $\begin{array}{r}2 / 22 \\
12 / 28\end{array}$ & $\begin{array}{l}4 / 18 \\
8 / 23\end{array}$ & $\begin{array}{l}\text { None } \\
10 / 28\end{array}$ & $88 \cdot 5$ & 100 \\
\hline
\end{tabular}

TABLE 6. Artificial challenge in progress with homologous and heterologous viruses

\begin{tabular}{|c|c|c|c|c|c|c|c|c|}
\hline \multirow[b]{2}{*}{ Study } & \multirow[b]{2}{*}{ Groups } & \multirow[b]{2}{*}{ Challenge virus } & \multirow[b]{2}{*}{ Illness } & \multirow[b]{2}{*}{$\begin{array}{l}\text { Virus } \\
\text { isolation }\end{array}$} & \multirow[b]{2}{*}{$\begin{array}{l}\text { Serum } \\
4 \mathrm{x} \text { rise }\end{array}$} & \multirow{2}{*}{$\begin{array}{l}\text { Virus } \\
\text { associated } \\
\text { illness }\end{array}$} & \multicolumn{2}{|c|}{ Protection rate $(\%)$} \\
\hline & & & & & & & Illness & $\begin{array}{c}\text { Virus } \\
\text { associated }\end{array}$ \\
\hline \multirow[t]{2}{*}{ Rytel } & 'Alice' & Udorn BEK4E1 & $1 / 12$ & $0 / 12$ & $0 / 12$ & $0 / 12$ & \multirow{2}{*}{$89 \cdot 6$} & \multirow{2}{*}{100} \\
\hline & Control & $\begin{array}{l}10^{6.12} \mathrm{EID}_{50} \\
(10 \text { months later })\end{array}$ & $8 / 10$ & $10 / 10$ & $8 / 10$ & $8 / 10$ & & \\
\hline \multirow[t]{2}{*}{ Waldman } & 'Alice' & Dunedin $/ 3 / 73$ & $0 / 6$ & $0 / 6$ & $0 / 6$ & $0 / 6$ & \multirow{2}{*}{100} & \multirow{2}{*}{100} \\
\hline & Control & $\begin{array}{l}10^{6.1} \text { EID }_{50} \\
( \pm 30 \text { days later })\end{array}$ & $3 / 7$ & $3 / 7$ & $6 / 6$ & $3 / 7$ & & \\
\hline
\end{tabular}

TABLE 7. Response of vaccinees to natural outbreak of 'Port Chalmers' influenza. Rochester, N.Y., March/April, 1974

\begin{tabular}{|c|c|c|c|c|c|c|}
\hline \multirow[b]{2}{*}{ Group } & \multirow[b]{2}{*}{$\begin{array}{c}\text { No. of } \\
\text { subjects }\end{array}$} & \multirow[b]{2}{*}{ No. ill } & \multicolumn{3}{|c|}{ No. infected } & \multirow{2}{*}{$\begin{array}{l}\text { Illness } \\
\text { associated } \\
\text { with } \\
\text { infection }\end{array}$} \\
\hline & & & $\begin{array}{l}\text { Virus } \\
\text { isolated }\end{array}$ & $\begin{array}{l}\text { Antibody } \\
\text { response }\end{array}$ & Total & \\
\hline $\begin{array}{l}\text { 'Alice' } \\
\text { Parenteral killed vaccine } \\
\text { No vaccine }\end{array}$ & $\begin{array}{l}45 \\
52 \\
14\end{array}$ & $\begin{aligned} 17 & (37 \cdot 8 \%) \\
20 & (38 \cdot 5 \%) \\
7 & (50 \%)\end{aligned}$ & $\begin{array}{ll} & 0 \\
5 & (9 \cdot 6 \%) \\
2 & (14 \cdot 3 \%)\end{array}$ & $\begin{array}{l}3(6 \cdot 7 \%) \\
5(9 \cdot 6 \%) \\
6(42 \cdot 9 \%)\end{array}$ & $\begin{array}{l}3(6 \cdot 7 \%)^{*} \\
8(15 \cdot 4 \%) \dagger \\
6(42 \cdot 9 \%) \dagger\end{array}$ & $\begin{array}{l}2(4 \cdot 4 \%) \\
7(13 \cdot 5 \%) \\
3(21 \cdot 4 \%)\end{array}$ \\
\hline
\end{tabular}

* Chi square, $P<0.05 ;+$ Chi square, $P<0.10>0.05$.

Protective efficacy

\begin{tabular}{lcc}
\hline \multicolumn{1}{c}{ Group } & Infection & Illness associated with infection \\
\hline 'Alice' & $84 \cdot 3 \%$ & $79 \cdot 4 \%$ \\
Killed parenteral vaccine & $64 \cdot 1 \%$ & $36 \cdot 9 \%$ \\
\hline
\end{tabular}

rate of infection was much lower among 'Alice' vaccinees and the difference with the control group was significant at the $5 \%$ level. Protection against infection was $84 \%$ in the 'Alice' vaccinees and $64 \%$ in the killed vaccine group. Protection against virusassociated illness was $79 \%$ and $37 \%$ for live and killed vaccines respectively. It is of interest to point out that seroconversion rates observed 5 weeks after vaccination (pre-challenge) were $40 \%$ for the live vaccine and $80 \%$ for the killed vaccine, confirming once more the lack of direct correlation between seroconversion rate and protection against challenge infection.

Table 8 summarizes the total experience in challenge studies with 'Alice' strain in man, with homologous (A/England/42/72) and heterologous (A/Port Chalmers/1/73) viruses, indicating the high degree of protection obtained with the 'Alice' strain. 
TABLE 8. Results from challenge studies in volunteers vaccinated with 'Alice' strain (2 doses)

\begin{tabular}{lccc}
\hline & $\begin{array}{c}\text { Incidence of } \\
\text { 'flu illness* }\end{array}$ & $\begin{array}{c}\text { Protection against } \\
\text { 'flu illness }\end{array}$ \\
\hline Homologous challenge A/England $/ 42 / 72$ & \\
Vaccinees & $0 / 34$ & $0 \%$ & $100 \%$ \\
Placebos & $18 / 38$ & $47 \cdot 36 \%$ & $100 \%$ \\
Heterologous challenge & A/Port Chalmers $/ 1 / 73$ \\
Vaccinees & $2 / 51$ & $3.92 \%$ & $86.28 \%$ \\
Placebos & $6 / 25$ & $28 \cdot 57 \%$ & \\
\hline
\end{tabular}

* Virus associated illness.

\section{Conclusion}

In conclusion, a live attenuated vaccine ('Alice' strain), administered intranasally, has been shown to provide a high degree of protection against challenge infection, both in an animal model and in man, and both with homologous and heterologous strains.

\section{Acknowledgments}

We wish to thank Drs R. G. Douglas (University of Rochester, New York), M. W. Rytel (Med. Coll. Wisc. Milwaukee), G. M. Schiff (Christ. Hosp. Inst. Med. Res., Cincinnati) and R. H. Waldman (University of Florida, Gainsville) for permission to use data obtained in their challenge studies.

\section{References}

Beare, A.S. (1969) Prospects for immunization against influenza by the use of live vaccines. Proceedings of the Royal Society of Medicine, 62, 47.

Beare, A.S., Hobson, D., Reed, S. \& Tyrrell, D.A. (1968) A comparison of live and killed influenza virus vaccines. L.ancet, ii, 418.

Huygelen, C., Peetermans, J., Vascoboinic, E., Berge, E. \& Colinet, G. (1973) Live attenuated influenza virus vaccine: in vitro and in vivo properties. International Symposium on Influenza Vaccines for Men and Horses. London 1972. Symposia Series in Immunobiological Standardization, 20, 152. Karger, Basel.

Lamy, F., Prinzie, A., Peetermans, J., Vascoboinic, E. \& Huygelen, C. (1973) Clinical evaluation of a live attenuated influenza virus vaccine ( $A n n$ strain). International Symposium on Influenza Vaccines for Men and Horses,
London 1972. Symposia Series in Immunobiological Sta dardization, 20, 282. Karger, Basel.

Mclarren, C., Potter, C.W. \& Jennings, R. (197\$ Immunity to influenza in ferrets. Journal of Hygiene \&f Cambridge, 73, 389.

ManN, J.J., Waldman, R.H., Togo, Y., Heiner, G.G DAWKINS, A.T. \& KASEL, J.A. (1968) Antibody respones in respiratory secretions of volunteers given live and def influenza virus. The Journal of Immunology, 100 (4), 80.

Noble, G.R., Rubin, R.J., Corey, L., Brandling-Bennet黑 D., Kaye, H.S., Coleman, M.T., Dowdle, W.R. \& Greg\& M.B. (1974) Attenuated influenza A/England/42/72 $\left(\mathrm{H}_{3} \mathrm{~N}_{\mathrm{g}}\right.$ vaccine. II. Serum hemagglutination inhibiting (HI) and neuraminidase inhibiting (NI) antibody responses. Four teenth Interscience Conference on Antimicrobial Agents and Chemotherapy, San Francisco, 11-13 September, 1974! Abstract no. 312. American Society for Microbiology.

Peetermans, J., Lamy, F. \& Delem, A. (1975) Immune $\$$ sponse to combined live influenza virus vaccines adminfo tered intranasally. International Symposium on Immuni to Infections of the Respiratory System in Man arid Animals, London 1974. Developments in Biologicat Standardization, 28, 340. Karger, Basel.

Peetermans, J., Prévost, J.M., Najera, R., Perez-Brein@̣, P. \& Huygelen, C. (1973) Quantitative and qualitatify antibody response to intranasal administration of a liea attenuated influenza virus vaccine. International Syno. posium on Influenza Vaccines for Men and Horses, London 1972. Symposia Series in Immunobiological Stañdardization, 20, 208. Karger, Basel.

Prévost, J.M., Vereerstraeten, J., Lamy, F. \& De Koste J.P. (1975) Live attenuated influenza virus vaccines in patients with chronic broncho-pulmonary diseases. ScIndp navian Journal of Respiratory Diseases, 56, 58.

Rubin, R.J., Noble, G.R., Corey, L., Brandling-BenNicic D., Kaye, H., Coleman, M.T., Brown, W.J., Dow甲י W.R. \& GREGG, M.B. (1974) Attenuated influenza्? England/42/72 $\left(\mathrm{H}_{3} \mathrm{~N}_{2}\right)$ vaccine. I. Virus transmission an adverse reactions. Fourteenth Interscience Conference Antimicrobial Agents and Chemotherapy, San Francisc 11-13 September 1974, Abstract no. 311. American Society for Microbiology.

Waldman, R.H., Lee, J.D., Lauteria, S.F. \& Kantzle G.B. (1975) An attenuated influenza virus vaccine: react $\sigma_{-}$ genicity, transmissibility, immunogenicity and protectiog efficacy. International Symposium on Immunity to Infee? tions of the Respiratory System in Man and Animass London 1974. Developments in Biological Standardizatiog 28, 347. Karger, Basel. 